\title{
A Computer-Based ICU Patient Alert And Decision Support System
}

\author{
Shashi Gunawardane ${ }^{1}$, Ravindra Koggalage ${ }^{2}$, Ranga Rodrigo ${ }^{3}$, Senaka Rajapakse ${ }^{4}$ \\ ${ }^{1}$ Faculty of Information Technology, University of Moratuwa, Sri Lanka, \\ ${ }^{2}$ The Associated Newspapers of Ceylon Ltd., Colombo, Sri Lanka, \\ ${ }^{3}$ Department of Electronics and Telecommunication, University of Moratuwa, Sri Lanka, \\ ${ }^{4}$ Department of Clinical Medicine, Faculty of Medicine, University of Colombo, Sri Lanka
}

\begin{abstract}
The safety of critically ill patients in intensive care units is an important aspect of medical care. Many human factors contribute to deficiencies and errors in patient care in the intensive care setting, such as long working hours, high levels of stress, lack of enough people, may cause human errors and affecting the effectiveness of the decisions of the physician. Several attempts have been made to increase the effectiveness of such decisions by issuing early alerts on adverse patient conditions. However, such alerts are based on single parameter variations, and not on the relationship between multiple parameter variations. We developed a computerbased model is an integrated solution which identifies adverse patient events based on multiple parameter variations, and then provides predictive treatment suggestions based on the likely clinical conditions which result in the parameter variations. The proposed system follows an interactive communication cycle in order to properly notify the responsible treating physicians at different tiers of responsibility. Our model is capable of early identification of adverse conditions and providing suitable treatment suggestions, thus acting as a decision support system to assist the treating physician.
\end{abstract}

\section{Introduction}

The safety of critically ill patients in intensive care units (ICUs) is an important aspect of medical care. In ICU, several parameters are measured in real time to monitor the progression of illness. These different parameters are all intricately linked to each other, each parameter affecting the other, and vice versa. These interactions are governed by complex but standard physiological principles, and are hence predictable.

Human error occurs all too frequently in patient care, especially in ICUs. Parameters or parameter trends which forewarn the development of serious adverse outcomes can be missed by the attending doctor or nurse. A variety of factors influence the occurrence of human error, such as the level of training and experience of the doctor/nurse, their attitudes and dedication towards work, whether the doctor/nurse is tired or overworked, lack of adequate staff, and also technical issues, such as delay in investigations reaching the bedside from the laboratory, and simple human error.
Computer based systems for continuous monitoring of biochemical and physiological parameters are currently available in many ICUs, especially in developing countries. Similarly, easy access to results of laboratory and radiological investigations is available through computer based information systems. These systems, however, simply provide discrete sets of information to the end user (the treating physician or nurse) and do not assist the user in the decision making process.

\section{Concept}

We set out to develop a model of a computer based automated alert and decision support system. This was undertaken as a joint project between the Faculty of Medicine, University of Colombo, and the University of Moratuwa.

Correspondence: Shashi Gunawardane,

Faculty of Information Technology,

University of Moratuwa, Sri Lanka.

Email: shash552@hotmail.com 
Our aim was to develop a system which could be fed with serial biochemical and physiological parameters about a patient, which would then be able to detect variations in parameters which would be potentially adverse. The system would then alert the treating physician or nurse regarding the abnormal parameter, and suggest a suitable intervention. Unlike simple high-low alert systems which are commonly used in ICU monitors, the system would analyze the values of multiple parameters, and the alert would occur based on a composite of all available parameters.

The presence of an automated decision system which will recognize both current adverse parameters as well as trends towards future adverse events will help minimize these errors. The proposed system focuses on the development of an automated decision support system which identifies important trends and adverse conditions based on the variations and the relationships between different parameters monitored. As stated earlier, these relationships are predictable; therefore the system would also able to suggest recommended treatment for the identified critical conditions. The system not only focuses on a decision making role but also on effective interaction between the system and physician by following a hierarchical communication model. This eliminates the human-computer interaction gap in most of the current ICU systems.

The criticality in patient conditions in an ICU environment has resulted in considerable research work with regard to intelligent decision making and and effective communication models. There are currently many systems which address the different aspects of medical decision making and some of them are currently being used in hospitals ${ }^{3,4}$.

In a decision support system, artificial intelligence can play a significant role in condition monitoring. One of the most common approaches in artificial intelligence is case-based reasoning ${ }^{5,6}$. Casebased reasoning provides a predetermined hierarchical structure which acts as a model for quickly matching a particular patient's parameters to a similar set of parameters contained in a clinical database. Such a comparison produces a group of people whose characteristics are exactly matched to the current patient characteristics. Casebased reasoning methodology attempts to produce a set of matches that are "as close as possible" to the current patient properties. Doctors may use case-based reasoning systems to find a group of the closest matching patients to their current patient and then the different aspects of the ICU history of the selected group such as the length of stay, mortality, hours of ventilation, procedures utilized, and complications encountered are graphically displayed, in order to analyze and make an effective decisions on current patient conditions. However, the ability to present real time predictive decisions is not supported by the system functionality. Therefore, this system is only useful at offline patient analysis but still at the expense of time of a medical physician.

Another extended version of health monitoring system is INTCare system 1 . It uses the agent-based paradigms for maintenance of the knowledge of an intelligent decision support system. This system is specially designed for the early detection of organ failure of six systems (liver, respiratory, cardiovascular, coagulation, central nervous and renal) and morality assessment. The system consists of four main sub systems: Data entry, knowledge management, inference, and interface. All the data acquisition activities are carried out by the Data Entry sub system which ensures the data is acquired in a such way that can be understood by the agent who responsible for knowledge maintenance. Knowledge management and inference sub systems basically consist of different types of agents who are designed to perform their role in a specific context. Several such agents are data mining agent, performance agent, model identification agent and inference agent. These agents are then put together to reason about the behavior of the entire system. Though the system is capable of maintaining dynamic knowledge, its knowledge specification is only limited to patient records. Therefore the system lacks in providing medical assistance on identified adverse conditions. 
Thus, the actual value of INTCare is reflected if and only if there is a dedicate physician to manage the system.

Neural networks based system uses a training set of patient data (text or numeric) to seek mathematical relationships between various subsets of patient parameters. Once the relationships are discovered, those are then applied to estimate the outcomes (e.g., mortality, length of stay, and hours of ventilation) of a new patients. For example, Buchman used a neural network to evaluate the relationship of several demographic, pharmacologic, and physiologic variables to ICU chronicity7. The neural nets are extensively used for predicting the critical conditions of ICU patients8. Apart from relationship identification and predictions, it also commonly used for real-time analysis of waveforms such as electrocardiogram and the electroencephalogram. Some such applications 2 are identification of cardiac ischemia with high sensitivity, analysis of electroencephalographic patterns in children and adults sedated with midazolam and analysis of hemodynamic patterns in intensive care patients. Even though neural nets play a major role in identifying the complex patterns, these systems would not be desirable for routine ICU monitoring since the results of neural nets are not often proved by explicit reasons. Therefore, a physician would not be able to reason out the critical conditions and provide appropriate treatments.

eICU9 and Patientrack10 are two ICU monitoring applications using currently in several hospitals. eICU is an attempt to monitor entire ICU environment from a single location. In addition, it provides patient data management tools and set of proprietary algorithms for alerting on critical patient conditions. Even though they claim their system is capable of identifying adverse patient conditions, it is questionable the accuracy and the scope of their algorithms. Patientrack is another alerting system developed with the minds of better communication model between the physicians. Both systems do not yet address the issue of identifying the relationships between different health parameters and thereby provide medical assistance on adverse patient conditions.

As highlighted earlier, the underlying objective of all the medical activities is to reliably understand, predict human health conditions, and thereby provide effective treatment. Where, computational technology can play a significant role in ensuring fast, reliable measurements in various aspects of medical domain.

The term 'interaction' is one of the important element in medical domain where the survival of the next moment of a patient is basically rely on a effective interaction model taking place within very short duration. This emphasizes the importance of interaction between every element who involves with any kind of medical activities. However, technological solutions have not yet realized importance of enabling an interactive machine-human environment. As a result most of sophisticated systems are used for output set of raw data, but not using their analytical power to retrieve meaningful information/results and effectively warn physicians about the patient conditions.

\section{Methods}

The proposed solution is to provide an interactive decision supporting system which basically produces decisions/recommendations for patient condition based on the values output by different health monitoring systems. This system will monitor the relationships and dependencies between different health parameters and provides next-step recommendations for doctors to carry out treatments on patient. The system keeps a hierarchy of responsible bodies and immediately alerts the responsible bodies about the negative conditions of patients and if the system does not receive any response from the desired physician, it will re-alert the next responsible physician in the hierarchy.

The important feature of this solution is its capability to identify different health condition patterns of the patient where the system will continuously monitor the health parameters over a period of time and any variations or adverse health 
patterns will be alerted. Thus, it will provide predictive decisions base on those identified patterns to allow doctors to make effective decisions. Once treatments are provided with the support of system recommendations, the system will closely monitor the effectiveness of such treatments and any adverse effect on different health parameters will immediately be notified.

The solution expects to overcome the interaction barrier between doctors and standalone health monitoring system by using expert knowledge in medical domain into a system, whereby, support effective decision making in a stressful surgery environment.

In technical terms, an expert system will be developed to represent knowledge of medical domain and pattern recognition techniques expected to be used for recognizing different patterns relevant to patient health conditions. These two modules will interact with each other to derive conclusions.

\section{System Architecture}

The system mainly takes inputs from the different health monitoring systems, analyze and store them in more user friendly manner. When a critical condition is identified, an interactive communication cycle is carried out with physician mobile phone in order to ensure that physician is properly informed about patient current conditions and suggested treatment $\mathrm{s} /$ he immediately required.

Figure 1 shows the proposed system design where the main components would be the trend analysis engine and the expert system. The trend analysis engine analyzes the variations of patient parameters while the expert system represents relationships between health parameters according to medical domain knowledge.

These two components will act together to identify adverse conditions and to suggest predictive medical treatments for such identified conditions. While these two component help in real-time decision making the Profiler will be used to non-realtime patient hidden characteristic identification by matching patient attributes with similar set of past patient attributes.
System controller can be considered as the internal communication bus of the system where it basically handles the interactions between user interfaces and the other internal system components. Profile manager is responsible for storing and managing the persistency of patient data. A short message service (SMS) gateway acts as the interface between system and mobile phone communication.

Figure 1. Component based architecture of the system with their interactions.

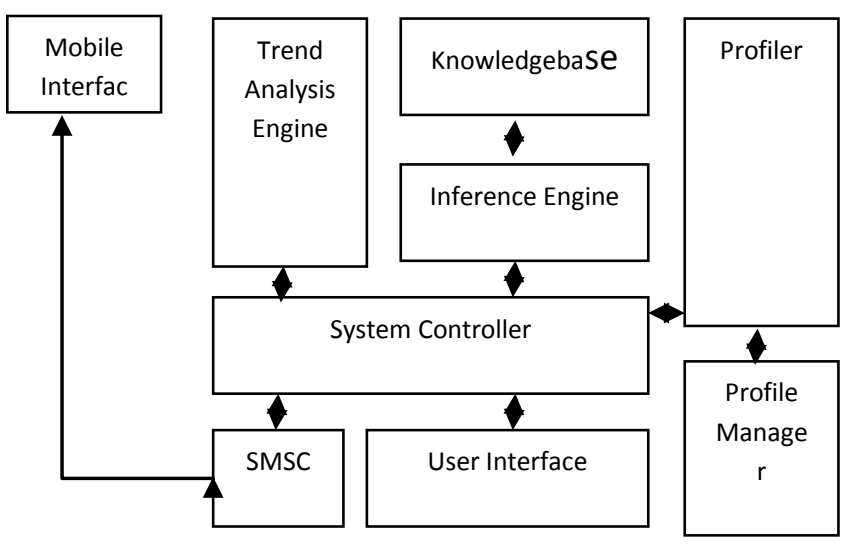

\section{Trend Analysis Engine}

Trend analysis engine basically consists of set of algorithms which are designed to identify different patterns based on the specific configuration. It is expected to monitor variations such as increasing and decreasing trends in short term and long term, seasonal variations and spikes in monitored data streams. There are number of statistical techniques available for analyzing parameter data sets with different accuracy levels and with different complexity. In general, a common set of parameters are usually monitoring on each ICU patient and there will be very few patient-specific parameters. Often, Medical experts make use of variations in those common set of parameters to conclude critical decisions on patient health. Those variations are varying from simple trends to more complex trends, however, in general, real time decision making is taking place using trends which are identifiable within the limitations of human analytical power. Therefore, it is expected use linear and nonlinear regression analysis for the identification of basic trends at the initial phase of the system, however, the module is 
designed in such a way that it could be easily extensible to perform much complex analysis.

The characteristics of the parameters that are being monitored such as their threshold values would vary depending on the nature of different human bodies. Therefore the trend analysis engine provides an interface for configuring parameter characteristics. This interface assists physician to monitor specific trends for each specific patient.

\section{Knowledge Representation}

Once a particular trend is identified by the Trend analysis engine, it is then need to identify whether the trend is a representation of a critical human condition which is defined by a combination of multiple parameter variations. Therefore it is necessary to have clear picture of relationships between those parameters in advance. The system is designed not only to alert at the identification of a critical condition but also to suggest medical treatments according to the conditions identified. Therefore it requires a proper mapping mechanism between patient conditions and the treatment suggestions.

In computer domain, parameter relationships can be viewed as a set of well defined rules while medical treatments can be consider as a set of facts which can be derived by satisfying different rules. At the implementation level, an expert system is expected to develop representing those rules and facts where backward chaining approach is followed. This approach, which is one of the strategies used to derive a conclusion, supports dynamic identification of defined relationships between rules by considering the values of individual rules. One of the logic programming languages called Prolog is selected for designing the rule based system since it supports the backward chaining approach. A simple rule set designed using Prolog language is shown in Figure 2, where the 'solution' template defines the conclusions/suggested treatments while 'parameter' templates define relationships between parameters to derive conclusions.
Fig. 2. Parameter relationships definitions using Prolog language

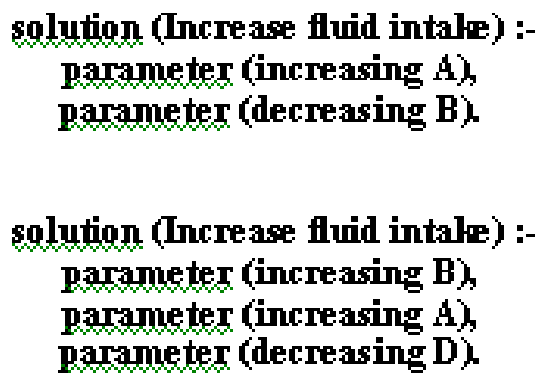

solution (Increase fluid intale) :parameter (increasing B), parameter (increasing A), parameter (decreasing D).

\section{Decision Making Model}

Figure 3 provides a detailed logical flow of interaction between Trend analysis engine and the Expert system in order make a successful decision. Once new data set is available for analysis, the Trend engine will process each of the parameter based on their priority level. If the Trend engine identifies an interested trend in a parameter it will forward it to the Expert system where it will try to match its rules with the forwarded trends. Generally, these rules consist of set of sub rules that need to be evaluated before approaching a conclusion. An example of such rule is shown in the Figure 3. According to the diagram, the trend "parameter $\mathrm{A}$ is increasing" is matched with a rule and expert system needs to know whether parameter B is also increasing. Therefore, it asks Trend engine to analyze the parameter $B$ and then Trend engine will analyze the parameter and inform results back to the Expert system. This interaction cycle is taking place until each of the sub rule is evaluated, then finally Expert system make the conclusion by taking into consideration all those evaluated results. Once the conclusion is made, then it will get the other parameter to be evaluated based on the priority level and reinitiate the above mentioned interaction process. In this way, all input parameters are evaluated. Finally, the system will compare all the conclusions, remove any duplicate conclusions and notify the relevant user interface and the mobile user. 
Figure 3. Interaction between trend engine and the rule based system in producing decisions on given parameter inputs.

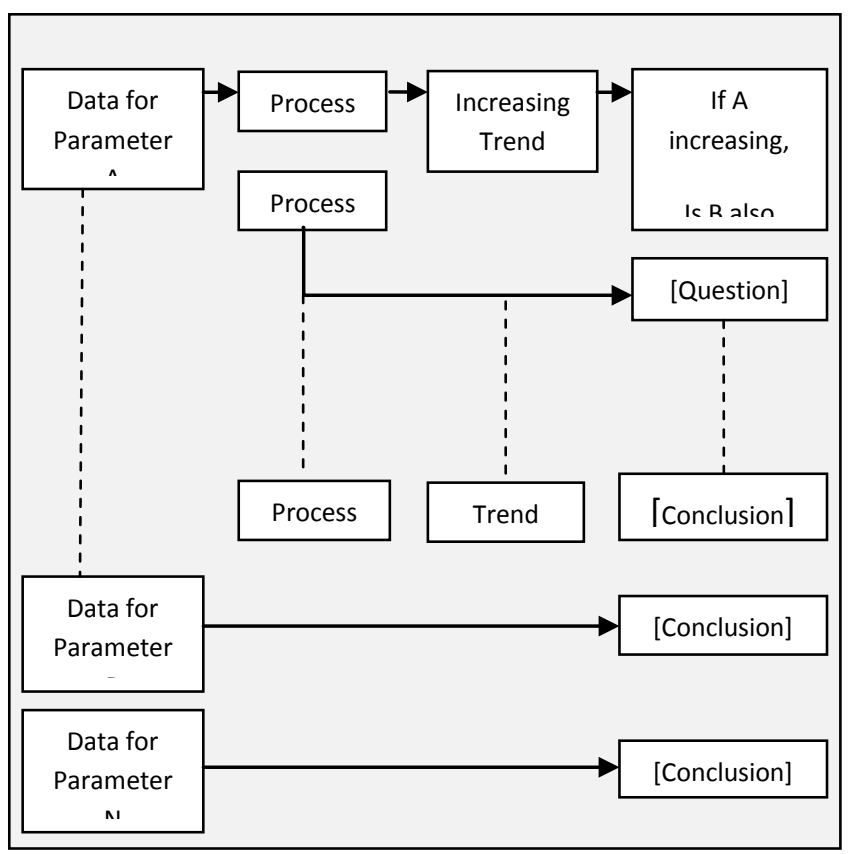

\section{Communication Model}

It is crucial to have an effective communication model in order to ensure the maximum usage of the system and is one of the factors lacks in most of the decision support systems. Most of the medical experts who involve with ICU operations are also involve with other nonfunctional hospital operations, therefore their availability in ICU environment is not always guaranteed. Therefore a proper communication model is important to alert the experts about the critical conditions of the patients in an informative way. This is achieved by keeping prioritized contact records of hierarchy of responsible physician for each patient and then forwarding the necessary information i.e. current parameter values, identified conditions, treatment suggested, to the mobile interface of the relevant physician by following the hierarchy. The physician will then able to immediately visit the patient and assign necessary treatments without further taking time to analyze the patient. On the other hand, if the physician is not in reachable distance to ICU unit, he would be able to transfer control of the patient to the next level of hierarchy via the mobile interface.

Tracking of treatments on patient based on the system alerts is one of the main features of the system where it will continuously monitor and generates different alerts if the subsequent treatments cause more adverse effect on patient conditions. This will help doctors to keep track of patient condition variations based on their treatments and react quickly on adverse patient reactions.

\section{Results}

In this section a comparison between system results and general physician decisions on a collected data sample is carried out in order to highlight at what stage the system would assist to the physician.

Table 1 depicts variations of set of parameters over a period of time (hourly) monitored on a particular patient. The rule based system consists of a rule shown in Figure 4. which defines the relationship between set of above monitored parameters and the set of conclusions and treatment suggestions that would prompt when such relationship is identified.

Generally, a physician would identify that the blood pressure of the patient is dropping only at the 17 th hour. Though, the proposed system identifies the above patient condition at the 11th hour by matching the variations of the pulse rate,

Table1 : Sample collected on a patient at icu

\begin{tabular}{|l|l|l|l|l|l|l|l|l|l|l|l|l|l|l|l|}
\hline Timeline (Hour) & $\mathbf{4}$ & $\mathbf{5}$ & $\mathbf{6}$ & $\mathbf{7}$ & $\mathbf{8}$ & $\mathbf{9}$ & $\mathbf{1 0}$ & $\mathbf{1 1}$ & $\mathbf{1 2}$ & $\mathbf{1 3}$ & $\mathbf{1 4}$ & $\mathbf{1 5}$ & $\mathbf{1 6}$ & $\mathbf{1 7}$ & $\mathbf{1 8}$ \\
\hline Systolic blood pressure & 130 & 135 & 110 & 100 & 90 & 90 & 90 & $\mathbf{8 5}$ & 85 & 85 & 80 & 80 & 70 & $\mathbf{7 0}$ & 100 \\
\hline Diastolic blood pressure & 90 & 85 & 80 & 75 & 70 & 60 & 60 & $\mathbf{6 0}$ & 50 & 50 & 50 & 50 & 50 & $\mathbf{5 0}$ & 50 \\
\hline Pulse rate & 80 & 85 & 90 & 110 & 120 & 120 & 130 & $\mathbf{1 3 0}$ & 130 & 130 & 130 & 130 & 130 & $\mathbf{1 3 0}$ & 130 \\
\hline Serum potassium & 4.5 & 4.5 & 4.5 & 5.0 & 5.5 & 5.5 & 5.5 & 5.8 & 5.9 & 6.5 & 6.5 & 6.6 & 6.8 & 6.8 & 6.2 \\
\hline
\end{tabular}


systolic blood pressure and the serum potassium level. Then it will immediately alert the patient condition to the responsible physician with the suggested treatment - increase fluid amount. This example demonstrates the system ability to identify the critical conditions at the early stage and thereby prevent the side effects on patient health due to late identification of critical conditions.

Figure 4. A rule stored in rule based system

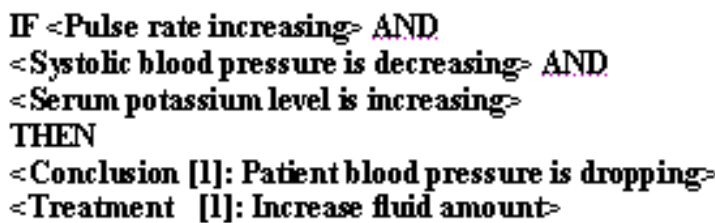

\section{Conclusion}

A patient monitoring and decision support system to overcome common problems in an ICU, including human error is proposed. The system is not intended to replace the medical experts but to provide a limited supportive role where the experts will be able identify patient condition variations more accurately and able to keep track of patient reactions to the treatments. Thus, the communication model of the system would allow medical experts to utilize their valuable time effectively while maximizing the ICU's operational efficiency.

The proposed system would be able to provide effective decision support in dealing with ICU patient conditions. However, the system functions based on a predefine set of rules and it would limit the identification of hidden relationships between parameters. Thus, the knowledge of the system is static and it needs to be continuously updated as new knowledge is discovered. This limitation is not addressed here and can be overcome by replacing the static rule-based system with a dynamic learning system which will itself learn the relationships between different parameters. Development of such a system is possible however it requires an enormous amount of data for training.

\section{References}

1. Paulo Cortez, Pedro Gago, Fernando Rua, Intelligent Decision Support in Intensive Care Medicine, Second International Conference on Knowledge Engineering and Decision Support, Lisbon, Portugal, pp 1-2, 2006.

2. C. William Hanson, Brayan E. Marshall, "Artificial Intelligance applications in intensive care unit", Crit Care Med Vol. 29, No. 2, 2001.

3. Advanced eICU Program Begins at HealthAlliance

Hospital,http://umassmemorial.org/He althAllianceIP.cfm?id=4411, June 8, 2008.

4. eICU Program Activates in Alaska, http://www.hospitaliteurope.com/defa ult.asp?page $=$ article.display\&title $=\mathrm{eICU}$ ProgramActivatesinAlaska\&article.id=1 5893, March 52009.

5. M. Frize, M. Stevenson, T. Buskard and K. Taylor, F.G. Solven and B. Nickerson, "Computer-assisted decision support systems for patient management in an incentive care unit", Medinfo'95, July, Vancouver, pp 1009-1012, July 1995.

6. M. Frize, H.C.E. Trigg, F.G. Solven, M. Stevenson and B.G. Nickerson, "Decision support systems designed for critical care", Proc AMIA Annu Fall Symp.,pp 855, 1997.

7. Buchman TG, Kubos KL, Seidler AJ and Siegforth MJ, "A comparison of statistical and connectionist models for the prediction of chronicity in a surgical intensive care unit",Crit Care Med 1994.

8. J. V. Tu and M. R. Guerriere, "Use of a neural network as a predictive instrument for length of stay in the intensive care unit following cardiac surgery", Proc Annu Symp Comput Appl Med Care.,pp 666-672, 1992.

9. eICU Integrated healthcare solution for critical care, http://www.visicu.com/products/index .html, Apr 27, 2009.

10. Pateintrack,http://www.patientrack.co m/OurSolution.php, May 3, 2009. 